\title{
Could there be a link between oral hygiene and the severity of SARS-CoV-2 infections?
}

\author{
Victoria Sampson, ${ }^{* 1}$ Nawar Kamona ${ }^{2}$ and Ariane Sampson ${ }^{3}$
}

\section{Key points}

Describes what COVID-19 is and the associated risk

Investigates whether bacteria contribute to COVID-19 complications by causing bacterial superinfections.
Examines whether there is a link between the oral microbiome and COVID-19 complications.

\begin{abstract}
On 30 January 2020, the World Health Organisation identified COVID-19, caused by the virus SARS-CoV-2, to be a global emergency. The risk factors already identified for developing complications from a COVID-19 infection are age, gender and comorbidities such as diabetes, hypertension, obesity and cardiovascular disease. These risk factors, however, do not account for the other $52 \%$ of deaths arising from COVID-19 in often seemingly healthy individuals. This paper investigates the potential link between SARS-CoV-2 and bacterial load, questioning whether bacteria may play a role in bacterial superinfections and complications such as pneumonia, acute respiratory distress syndrome and sepsis. The connection between COVID-19 complications and oral health and periodontal disease is also examined, as the comorbidities at highest risk of COVID-19 complications also cause imbalances in the oral microbiome and increase the risk of periodontal disease. We explore the connection between high bacterial load in the mouth and post-viral complications, and how improving oral health may reduce the risk of complications from COVID-19.
\end{abstract}

\section{Introduction}

On 31 December 2019, the World Health Organisation (WHO) was informed of a cluster of 27 cases of pneumonia with no known cause linked to a wet animal wholesale market in Wuhan City, China. ${ }^{1}$ By 7 January 2020, the causative virus was identified as SARS-CoV-2, which causes the disease COVID-19, and was declared a global emergency by the WHO on 30 January 2020.

Zhu et al. (2020) were able to identify and characterise SARS-CoV-2 using unbiased sequencing samples from infected patients with pneumonia. ${ }^{2}$ The viral genome revealed SARS-CoV-2 to be $75-80 \%$ identical to severe acute respiratory syndrome coronavirus

'Dentistry, 38 Devonshire Street, London, W1G 6QB, UK ${ }^{2}$ Centre for Nutrition Education \& Lifestyle Management, London, RG40 1DH, UK; ${ }^{3}$ Orthodontics, Cambridge

University Hospital Trust, Cambridge, CB1 00Q, UK.

${ }^{*}$ Correspondence to: Victoria Sampson

Email address: drvictoriasampson@hotmail.com

Refereed Paper.

Accepted 21 May 2020

https://doi.org/10.1038/s41415-020-1747-8
(SARS-CoV) and several bat coronaviruses. Prior to this discovery, six coronaviruses were known to cause human disease. Four viruses (229E, OC43, NL63 and HKU1) are prevalent and cause common cold symptoms. The two other strains are SARS-CoV and Middle East respiratory syndrome Coronavirus (MERS$\mathrm{CoV})$, both of which are zoonotic in origin and fatal. SARS-CoV-2 has joined as the seventh member of the family of coronaviruses that infect humans. ${ }^{2}$ No specific treatment is yet accessible; however, management involves restriction of travel, patient isolation and the support of medical supervision. ${ }^{3}$

COVID-19 affects people in different ways, with patients exhibiting a range of symptoms and severity. While risk factors such as age, gender and comorbidities have been highlighted as increasing the risk of complications and mortality, there is still a high proportion of patients with no identified risk factors who suffer from severe side effects and complications. As much as $10-15 \%$ of people under 60 years old with no risk factors are exhibiting moderate to severe reactions to COVID-19. ${ }^{1}$ While COVID-19 has a viral origin, it is suspected that in severe cases, bacterial superinfections may contribute to causing complications such as pneumonia and acute respiratory distress syndrome (ARDS). We explore the connection between high bacterial load in the mouth and postviral complications, and how improving oral health may reduce the risk of complications from COVID-19.

\section{Symptoms}

The clinical symptoms of COVID-19 appear after an incubation period of approximately 5.2 days $^{4}$ and range from fever (98.6\%), fatigue (69.6\%), dry cough (59.4\%), myalgia (34.8\%) and sore throat (17.4\%). ${ }^{5}$ Diarrhoea has also been shown to be a key distinguishing symptom of COVID-19 compared with SARS-CoV and MERS-CoV. ${ }^{6,7}$

COVID-19 infections can present with mild, moderate or severe illness. The severity of the illness, the rate of decline and the risk of mortality are significantly dependent on risk factors highlighted by Zhou et al. (2020) in the largest retrospective cohort study among 
patients with COVID-19. ${ }^{8}$ These risk factors include age (the mean age was 69 years old), gender (men represented $70 \%$ of deaths) and an underlying comorbidity in $48 \%$ of cases (hypertension $30 \%$, diabetes $19 \%$, or heart disease 8\%). In April, research from Europe added obesity as a risk factor for developing complications from COVID-19, with $47.6 \%$ of patients in intensive care units (ICUs) having a BMI over $30 \mathrm{~kg} / \mathrm{m}^{2} .{ }^{9}$ The main complications of severe illness are blood clots, pneumonia, sepsis, septic shock and ARDS. ${ }^{1}$ It has also been discovered that, in some cases, a dysregulated immune reaction triggers overproduction of early response pro-inflammatory cytokines (tumour necrosis factor [TNF], IL-6, and IL-1 $\beta$ ), resulting in a 'cytokine storm. ${ }^{10}$ This exposes patients to increased risk of vascular hyperpermeability and multi-organ failure, particularly to the heart and kidneys. ${ }^{11,12,13}$ IL-6 levels are shown to be comparatively higher in non-survivors than those who survive. ${ }^{10}$

When pneumonia spreads to the lungs and blood oxygen levels fall, patients require assisted ventilation, shifting their diagnosis from pneumonia to ARDS. Of 201 severely affected inpatients in China, $41.8 \%$ developed ARDS. Of these, $52.4 \%$ died due to respiratory failure, making ARDS the primary cause of death in infected patients. ${ }^{14}$ This is supported by a country report covering Italy, showing that $96.5 \%$ of complications arising from COVID-19 infection were ARDS, followed by acute renal failure (29.2\%). ${ }^{15}$ This suggests that patients are more likely to die from post-viral infection complications than from COVID-19. ARDS was also the biggest cause of death for SARS-CoV ${ }^{16}$ and MERS-CoV infections, ${ }^{17}$ further confirming the connection between the three coronaviruses. As of 3 March 2020, COVID-19 was predicted to have a $3.4 \%$ mortality rate. ${ }^{5}$ The period from the onset of symptoms to death averages 14 days.

\section{Discussion}

A significant unanswered question is why some patients suffer from COVID-19 more severely than others. Though patients with risk factors such as age, gender and comorbidities have an increased rate of complications and mortality, there is still a high proportion of seemingly young and healthy infected patients with no identified risk factors who suffer from severe side effects and complications, while some infected patients never go on to develop anything more than anosmia. ${ }^{18}$ It is common

Fig. 1 The different outcomes of different treatment options for COVID-19 patients. Reproduced from Gautret P, Lagier J-C, Parola P et al., Hydroxychloroquine and azithromycin as a treatment of COVID-19: results of an open-label non-randomized clinical trial, Int J Antimicrob Agents, 2020, Elsevier ${ }^{23}$

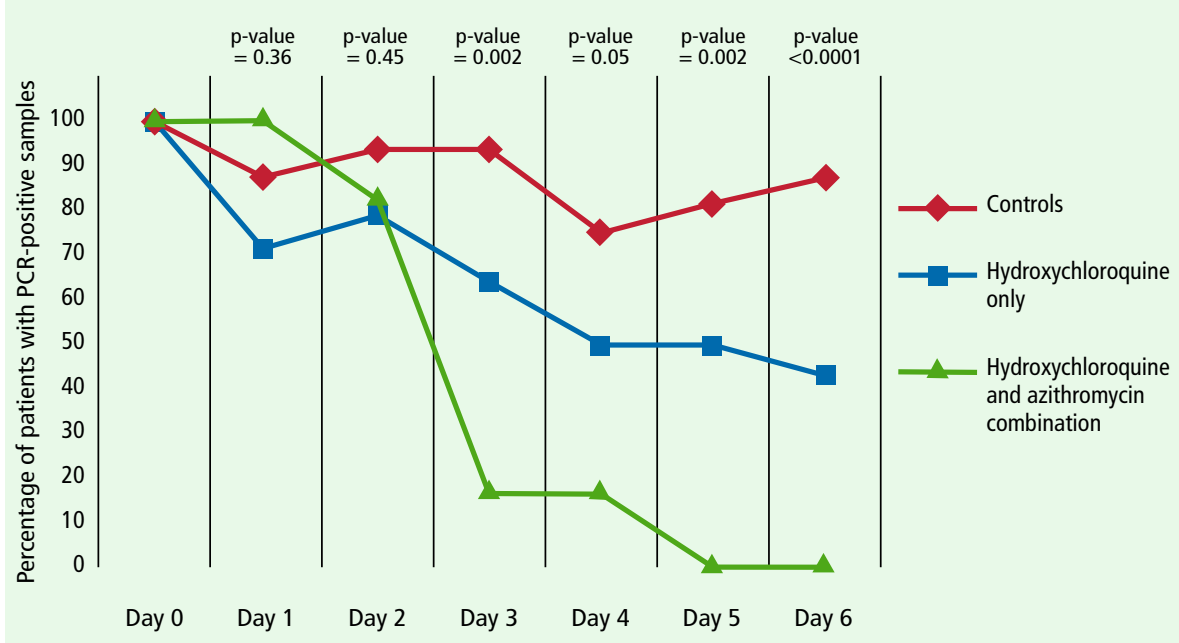

for respiratory viral infections to predispose patients to bacterial superinfections, leading to increased disease severity and mortality; for example, during the influenza pandemic in 1918, where the primary cause of death was not from the virus itself but from bacterial superinfections. The same was apparent in the $2009 \mathrm{H} 1 \mathrm{~N} 1$ influenza pandemic, where again bacterial superinfections were the primary cause of death as opposed to the virus itself. ${ }^{19}$ Despite the proven importance of superinfections in the severity of respiratory disease, they are often understudied during respiratory infection outbreaks, mainly due to the fact that diagnosing a superinfection is complex. $^{19}$

Other strands of the coronavirus have shown to enhance streptococcal adherence to epithelial cells along the respiratory tract, causing complications such as pneumonia ${ }^{20}$ and inflammatory damage in the lungs that inhibit the clearance of bacteria. Zheng et al. ${ }^{21}$ compared the levels of granulocytes in patients suffering from mild COVID-19 infections to those suffering from severe infections. Patients suffering from severe infections had a remarkably higher neutrophil count and a significantly lower lymphocyte count than in mild patients. A high neutrophil count is abnormal for a viral infection, but very common for a bacterial infection, resulting in Zheng et al. suspecting that, in severe cases of COVID-19, bacterial superinfection is common. Zheng et al. argued that abnormally low levels of lymphocytes, which are usually the main line of defence against viral infections, indicates either functional exhaustion of cells ${ }^{21}$ or potentially that, in severe cases of the disease, a bacterial superinfection supersedes the original viral infection. This is supported by the fact that Zhou et al. noted $50 \%$ of patients with severe COVID-19 died with the presence of a secondary bacterial infection.

When lymphocyte levels reduce, severity increases. This was also seen by Liu et al., ${ }^{22}$ who deduced that the neutrophil-tolymphocyte ratio predicts the severity of the patient's response to COVID-19. They found that in severe cases, over $80 \%$ of patients had an exceptionally high bacterial load secondary to a bacterial superinfection and required antibiotics. This is supported by the promising research performed in France, ${ }^{23}$ where a combination of hydroxychloroquine (antiviral) and azithromycin (antibiotic) was shown to cure $100 \%$ of patients virologically after six days, compared to patients who had hydroxychloroquine alone (57.1\%) and those who had no treatment $(12.5 \%)$. While the use of hydroxychloroquine has been under much dispute, it is important to understand that antibiotics clearly play an influential role in treatment outcome, improving the success rate by $42.9 \%$ in this case. A retrospective report of 1,060 patients in May 2020 confirms the efficacy of using a combination of an antibiotic with an antiviral, with $91.7 \%$ virologically cured after ten days. ${ }^{24}$ Furthermore, Chen 
et al. reported that $71 \%$ of COVID-19 patients in China needed antibiotics upon admission to hospital, ${ }^{25}$ while in Italy, $84 \%$ of patients needed antibiotics, ${ }^{26}$ illustrating the potential importance of antibiotics and therefore bacteria in the course of a COVID-19 complication. ${ }^{19}$ See Figure 1 for the outcomes of different treatment options for COVID-19.

The lungs, similar to the oral cavity, are often referred to as an 'ecological community of commensal, symbiotic and pathogenic organisms.'. ${ }^{27}$ Microbial immigration and elimination are constant between the oral cavity and the lungs, allowing for a healthy microbiotic distribution. ${ }^{28}$ Similar to the gut or the oral cavity, bacterial communities that colonise the lungs are recognised for their function in conserving tissue, immune and organ homeostasis. The lungs are unique, however, as they are oxygen-rich and therefore contain a fine equilibrium of lipidrich surfactants that influence bacteriostatic activity. This equilibrium can alter drastically during illness, allowing microbial overgrowth and injury to the lungs. Lower respiratory infection is initiated by the contamination of the lower airway epithelium by inhalation of microorganisms encompassed in aerosolised droplets, or by aspiration of oral secretions associated with oral disease (containing microorganisms such as $\mathrm{P}$. gingivalis, $\mathrm{F}$. nucleatum, P. intermedia).$^{29}$ Periodontitis and decay are the two most common oral diseases associated with an imbalance of pathological bacteria in the mouth. Cytokines (such as IL-1 and TNF) from periodontally diseased tissues can infiltrate the saliva through the gingival crevicular fluid and be aspirated to cause inflammation or infection within the lungs (Fig. 2) ${ }^{30}$ Therefore, inadequate oral hygiene can increase the risk of inter-bacterial exchanges between the lungs and the mouth, increasing the risk of respiratory infections and potentially post-viral bacterial complications. ${ }^{31}$ In a nationwide population-based cohort study, 49,400 chronic periodontitis patients were treated with periodontal therapy over 11 years. The Kaplan-Meier plot demonstrated the total incidences of pneumonia significantly decreased over a 12-year follow-up in the group receiving periodontal therapy $(\mathrm{p}<0.001)$. The reduction or eradication of periodontal disease significantly reduces one's risk of pneumonia.

Good oral hygiene has been recognised as a means to prevent airway infections in patients, especially in those over the age of $70 .{ }^{32}$ The oral cavity houses more than 700 bacteria, viruses

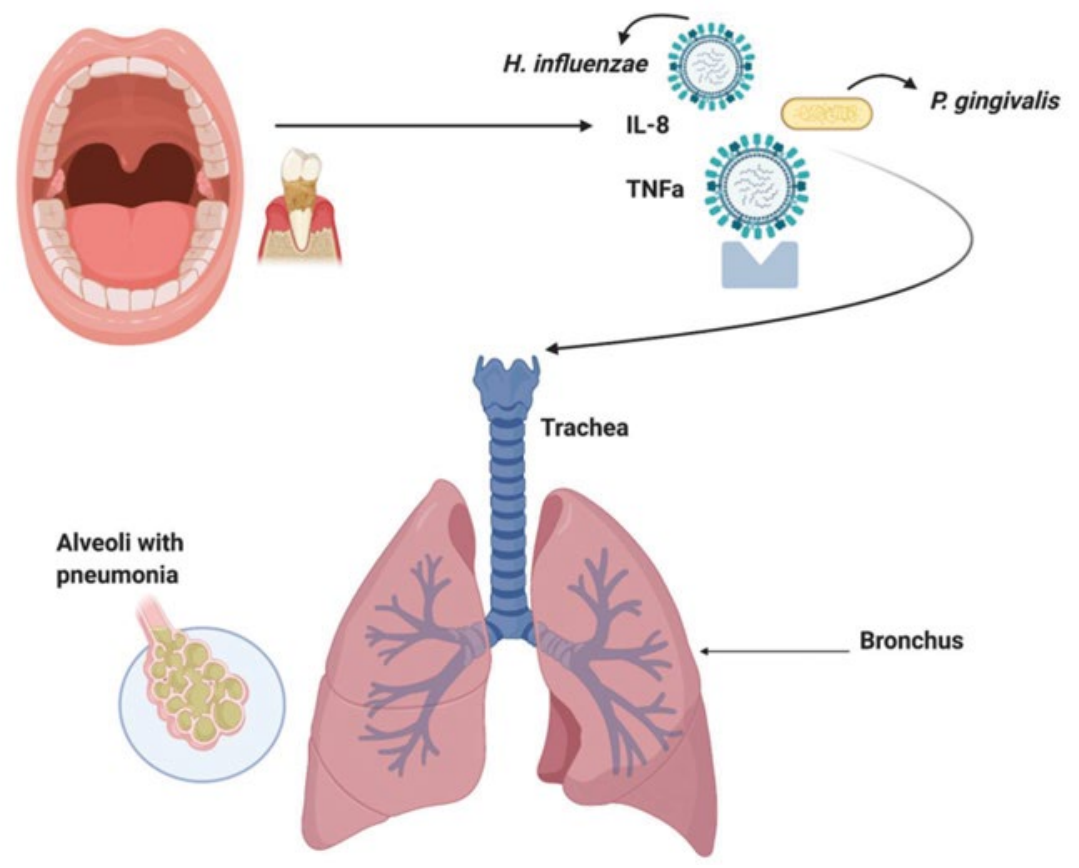

Fig. 2 Bacteria that colonise the mouth are shed into the saliva. The pathogenic bacteria within the saliva can then be aspirated into the lower respiratory tract and cause or aggravate an infection. Reproduced with permission from Scannapieco $F$ A, Role of Oral Bacteria in Respiratory Infection, J Periodontol, 1999, John Wiley and Sons. ${ }^{30}$

and fungi that can colonise the mouth. ${ }^{33}$ Various microbiological habitats exist within the mouth; however, the primary bacterial inhabitants are P. intermedia, S. mutans, F. nucleatum and P. gingivalis. ${ }^{34}$

Several mechanisms have been proposed to explain the potential role of oral bacteria in the pathogenesis of a respiratory infection: ${ }^{30}$

1. Aspiration of oral pathogens into the lungs

2. Periodontal disease-associated enzymes may modify the mucosal surfaces to allow for adhesion and colonisation of respiratory pathogens

3. Periodontal disease-associated enzymes may destroy the salivary pellicles on bacteria to hinder clearance from mucosal surfaces

4. Respiratory epithelium may be altered by periodontal-associated cytokines to promote infection by respiratory pathogens. ${ }^{35}$

The bacteria in the oral biofilm are in prime position to be aspirated into the respiratory tract and help initiate or progress conditions such as pneumonia or sepsis. A high bacterial and viral load in the mouth can further complicate systemic diseases such as cardiovascular disease, cancer, neurodegenerative disease and autoimmune diseases, ${ }^{36}$ further supporting the link between mouth and body. The established risk factors for COVID-19 (age, gender and comorbidities $)^{8}$ are also heavily implicated in imbalances in the oral microbiome. Diabetes, hypertension and heart disease are associated with higher numbers of $\mathrm{F}$. nucleatum, P. intermedia and P. gingivalis, ${ }^{37}$ and associated with the progression of periodontal disease. Patients with periodontal disease are at a $25 \%$ increased risk of cardiovascular disease, ${ }^{38,39}$ triple the risk of diabetes mellitus ${ }^{40}$ and a $20 \%$ increased risk of hypertension. ${ }^{41}$ Epithelial sensitisation and haematogenous spread of proinflammatory mediators such as cytokines, produced in the diseased periodontal tissue, can increase systemic inflammation and diminish airflow. This can be exacerbated by the stimulation of the liver to produce acute phase proteins, such as interleukin-6, which potentiate the inflammatory response of the lungs and the rest of the body. ${ }^{42}$ Similarly, patients with severe COVID-19 infections also express systemic inflammation and significantly higher levels of interleukin-6, interleukin-2, interleukin-10, TNF and C-reactive protein. ${ }^{43}$

Nagaoka et al..$^{44}$ investigated the effects of periodontopathic bacteria such as $\mathrm{F}$. nucleatum, P. gingivalis and P. intermedia on pneumonia, and found that P. intermedia in particular induced severe pneumonia in subjects with higher levels of periodontopathic bacteria. Pneumonia and acute viral 
respiratory infections are two of the most common airway infections in older patients ${ }^{45}$ and the greatest causes of death in patients over 70 years. A randomised controlled trial in Japan investigated whether improved oral care reduced the incidence of pneumonia and pneumonia-related deaths. Four hundred and seventeen patients were provided with oral care after every meal and compared with the control group. Of the control group, 19\% contracted pneumonia compared with only $11 \%$ who received oral care. Moreover, the postpneumonia mortality rate in the control group was almost double that of the group prescribed oral care. ${ }^{45}$ The link between good oral care and a reduced risk of acute viral respiratory infections has been established in a number of other studies, ${ }^{30,46,47,48}$ including a systematic review that concluded one in ten pneumoniarelated deaths in the elderly could be prevented by improving oral hygiene. ${ }^{32}$ Furthermore, improved oral care can significantly reduce the incidence of ventilator-associated pneumonia in ICU patients. ${ }^{49}$

\section{Conclusion}

The more severe the form of COVID-19, the higher the chance of complications such as pneumonia, ARDS, sepsis, septic shock and death. The development, severity and risk of complications following a COVID-19 infection depend on a number of host and viral factors that will affect a patient's immune response. While $80 \%$ of patients with COVID-19 infections have mild symptoms, $20 \%$ progress to have a severe form of infection associated with higher levels of inflammatory markers (IL-2, IL-6, IL-10), bacteria and neutrophil-to-lymphocyte count.

We suggest that the connection between the oral microbiome and COVID-19 complications should be investigated in the process of better understanding the outcomes of COVID-19 disease. The four main comorbidities associated with an increased risk of complications and death from COVID-19 are also associated with altered oral biofilms and periodontal disease, hence why the link between poor oral health and COVID-19 complications is suggested. Periodontopathic bacteria are implicated in systemic inflammation, bacteraemia, pneumonia and even death. Periodontopathic bacteria is also present in the metagenome of patients severely infected with SARS-CoV-2, where high reads for Prevotella (493 reads), Staphylococcus (1,659 reads) and
Fusobacterium (463 reads) were discovered. ${ }^{50}$ It is clear that bacterial superinfections are common in patients suffering from a severe case of COVID-19, with more than $50 \%$ of deaths exhibiting bacterial superinfections. ${ }^{19}$ Furthermore, it is common for respiratory viruses to predispose patients to bacterial superinfections, as seen in the influenza outbreaks in 1918 and $2009 .{ }^{19}$ Over $80 \%$ of patients in ICU exhibited an exceptionally high bacterial load, ${ }^{22}$ and treatment has been successful with a dual regime of an antiviral and an antibiotic. ${ }^{23}$

Despite the proven importance of superinfections in the severity of respiratory viral diseases, they are often understudied due to the complexity of diagnosis and culturebased microbiological testing being less sensitive once antibiotics are administered. ${ }^{19}$

More research should be performed on bacterial superinfections, and the connection, if any, between the oral microbiome and COVID-19 complications are urgently required to establish the importance of oral hygiene and pre-existing oral disease in the severity and mortality risk of COVID-19.

Meanwhile, we recommend that oral hygiene be maintained, if not improved, during a SARS-CoV-2 infection in order to reduce the bacterial load in the mouth and the potential risk of a bacterial superinfection. We recommend that poor oral hygiene be considered a risk to post-viral complications, particularly in patients already predisposed to altered biofilms due to diabetes, hypertension or cardiovascular disease. Bacteria present in patients with severe COVID-19 are associated with the oral cavity and improved oral hygiene may play a part in reducing the risk of complications.

\section{Conflict of interest}

The authors declare no conflicts of interest related to the study.

\section{References}

1. World Health Organisation. Clinical Management of severe acute respiratory infection when novel coronavirus (2019-nCoV) infection is suspected. 2020. Available online at https://apps.who.int/iris/ handle/10665/330893 (accessed June 2020).

2. Zhu N, Zhang D, Wang W. A novel coronavirus from patients with pneumonia in China. N Eng/ J Med 2020; 382: 727-733.

3. Zhang H, Penninger J M, Li Y, Zhong N, Slutsky A S. Angiotensin-converting enzyme 2 (ACE2) as a SARSCoV2 receptor: molecular mechanisms and potential therapeutic target. Intensive Care Med 2020; 46: 586-590.

4. Li Q, Guan X, Wu P et al. Early transmission dynamics in wuhan, China, of novel coronavirus infected pneumonia. N Engl J Med 2020; 382: 1199-1207.

5. World Health Organisation. Coronavirus disease (COVID19) pandemic. 2020. Available online at https://www. who.int/emergencies/diseases/novel-coronavirus-2019 (accessed June 2020).

6. Rothan H A, Byrareddy S N. The epidemiology and pathogenesis of coronavirus disease (COVID-19) outbreak. J Autoimmun 2020; DOI: 10.1016/j. jaut.2020.102433.

7. Greenland J R, Michelow M D, Wang L, London M J. COVID 19 Infection: Implications for Perioperative and Critical Care Physicians. Anesthesiology 2020; 132: 1346-1361.

8. Zhou F, Yu T, Du R et al. Clinical course and risk factors for mortality of adult inpatients with COVID-19 in Wuhan, China: a retrospectove study. Lancet 2020; 395: 1054-1062.

9. Simonnet A, Chetboun M, Poissy J et al. High prevalence of obesity in severe acute respiratory syndrome coronavirus-2 (SARSCoV2) requiring invasive mechanical ventilation. Obesity (Silver Spring) 2020; DOI: 10.1002/ oby. 22831.

10. Tay M Z, Poh C M, Rénia L, MacAry P A, Ng L F P. The Trinity of COVID-19: Immunity, Inflammation and Intervention. Nat Rev Immunol 2020; 20: 363-374.

11. Jose R J, Manuel A. COVID-19 cytokine storm: the interplay between inflammation and coagulation. Lancet Respir Med 2020; DOI: 10.1016/ S2213-2600(20)30216-2.

12. Perico L, Benigni A, Remuzzi G. Should COVID-19 Concern Nephrologists? Why and to What Extent? The Emerging Impasse of Angiotensin Blockade. Nephron 2020; 144: 213-221.

13. Yao X H, Li T Y, He Z C et al. A pathological report of three COVID-19 cases by minimally invasive autopsies. Chin J Pathol 2020; 49: 411-417.

14. Wu C, Chen X, Cai Y et al. Risk Factors Associated With Acute Respiratory Distress Syndrome and Death in Patients With Coronavirus Disease 2019 Pneumonia in Wuhan, China. JAMA Intern Med 2020; DOI: 10.1001/ jamainternmed.2020.0994.

15. European Centre for Disease Prevention and Control. Coronavirus disease 2019 (COVID-19) pandemic: increased transmission in the EU/EEA and the UK seventh update. 2020. Available at https://www.ecdc europa.eu/sites/default/files/documents/RRA-seventhupdate-Outbreak-of-coronavirus-disease-COVID-19.pdf (accessed June 2020).

16. Rubenfeld G D. Is SARS just ARDS? JAMA 2003; 290: 397-399.

17. Li X, Geng M, Peng Y, Meng L, Lu S. Molecular immune pathogenesis and diagnosis of COVID-19. J Pharm Anal 2020; 10: 102-108.

18. Shi Y, Wang Y, Shao C et al. COVID-19 infection: the perspectives on immune responses. Cell Death Differ 2020; 27: 1451-1454.

19. Cox M J, Loman N, Bogaert D, O'Grady J. Co-infections: potentially lethal and unexplored in COVID-19. Lancet 2020; DOI: 10.1016/S2666-5247(20)30009-4.

20. Golda A, Malek N, Dudek B et al. Infection with human coronavirus NL63 enhances streptococcal adherence to epithelial cells. J Gen Virol 2011; 92: 1358-1368.

21. Zheng $M, G a o Y$, Wang $G$ et al. Functional exhaustion of antiviral lymphocytes in COVID-19 patients. Cell Mol Immunol 2020: 17: 533-535.

22. Liu J, Liu Y, Xiang P et al. Neutrophil to Lymphocyte Ratio Predicts Critical Illness Patients with 2019 Coronavirus Disease in the Early Stage. J Trans/ Med 2020; 18: 206.

23. Gautret P, Lagier J-C, Parola P et al. Hydroxychloroquine and azithromycin as a treatment of COVID-19: results of an open-label non-randomized clinical trial. Int J Antimicrob Agents 2020; DOI: 10.1016/j. ijantimicag.2020.105949.

24. Million M, Lagier J-C, Gautret P et al. Early Treatment of COVID-19 Patients With Hydroxychloroquine and Azithromycin: A Retrospective Analysis of 1061 Cases in Marseille, France. Travel Med Infect Dis 2020; DOI: 10.1016/j.tmaid.2020.101738.

25. Chen T, Wu D, Chen $\mathrm{H}$ et al. Clinical characteristics of 113 deceased patients with coronavirus disease 2019: retrospective study. BMJ 2020; DOI: 10.1136/bmj. m1091.

26. Sorbello M, El-Boghdadly K, Di Giacinto I et al. The Italian Coronavirus Disease 2019 Outbreak: Recommendations From Clinical Practice. Anaesthesia 2020; 75: 724-732. 
27. Toraldo D M, Conte L. Influence of the Lung Microbiota Dysbiosis in Chronic Obstructive Pulmonary Disease Exacerbations: The Controversial Use of Corticosteroid and Antibiotic Treatments and the Role of Eosinophils as a Disease Marker. J Clin Med Res 2019; 11: 667-675.

28. Mathieu E, Escribano-Vazquez U, Descamps D et al. Paradigms of Lung Microbiota Functions in Health and Disease, Particularly, in Asthma. Front Physio/ 2018; DOI: 10.3389/fphys.2018.01168.

29. Scannapieco F A, Genco R J. Association of periodontal infections with atherosclerotic and pulmonary diseases. J Periodontal Res 1999; 34: 340-345.

30. Scannapieco F A. Role of Oral Bacteria in Respiratory Infection. J Periodontol 1999; 70: 793-802.

31. Paju S, Scannapieco F A. Oral biofilms, periodontitis, and pulmonary infections. Oral Dis 2007; 13: 508-512.

32. Sjögren $P$, Nilsson $E$, Forsell $M$, Johansson $O$, Hoogstraate J. A systematic review of the preventative effect of oral hygiene on pneumonia and respiratory tract infection in elderly people in hospitals and nursing homes: effect estimates and methodological quality of randomised controlled trials. J Am Geriatr Soc 2008; 56: 2124-2130.

33. Aas J A, Paster B J, Stokes L N, Olsen I, Dewhirst F E. Defining the normal bacterial flora of the oral cavity. J Clin Microbiol 2005; 43: 5721-5732.

34. Huffnagle G B, Dickson R P, Lukacs N W. The respiratory tract microbiome and lung inflammation: a two-way street. Mucosal Immunol 2017; 10: 299-306.

35. Hayata M, Watanabe N, Tamura M. The Periodontopathic Bacterium Fusobacterium nucleatum
Induced Proinflammatory Cytokine Production by Human Respiratory Epithelial Cell Lines and in the Lower Respiratory Organs in Mice. Cell Physiol Biochem 2019; 53: 49-61.

36. Olsen I, Yamazaki K. Can oral bacteria affect the microbiome of the gut? J Oral Microbiol 2019; DOI: 10.1080/20002297.2019.1586422

37. Takahashi K, Nishimura F, Kurihara M et al. Subgingival microflora and antibody responses against periodontal bacteria of young Japanese patients with type 1 diabetes mellitus. J Int Acad Periodontol 2001; 3: 104-111.

38. DeStefano F, Anda R F, Kahn H S, Williamson D F, Russell C M. Dental disease and the risk of coronary heart disease and mortality. BMJ 1993; 306: 688-691.

39. Paizan M L M, Vilela-Martin J F. Is There an Association between Periodontitis and Hypertension? Curr Cardiol Rev 2014; 10: 355-361.

40. Preshaw P M, Alba A L, Herrera D et al. Periodontitis and diabetes: a two-way relationship. Diabetologia 2012; 55: 21-31.

41. Aguilera E M, Suvan J, Buti J et al. Periodontitis is associated with hypertension: a systematic review and meta-analysis. Cardiovasc Res 2020; 116: 28-39.

42. Gomes-Filho I S, Seixas da Cruz S, Trindade S C et al. Periodontitis and respiratory diseases: a systematic review with meta-analysis. Oral Dis 2020; 26: 439-446.

43. Gong J, Dong H, Xia S Q et al. Correlation Analysis Between Disease Severity and Inflammation-related Parameters in Patients with COVID-19 Pneumonia.
2020. Available online at https://www.medrxiv.org/ content/10.1101/2020.02.25.20025643v1 (accessed June 2020).

44. Nagaoka K, Yanagihara K, Morinaga Y et al. Prevotella intermedia Induces Severe Bacteraemic Pneumococcal Pneumonia in Mice with Upregulated PlateletActivating Factor Receptor Expression. Infect Immun 2014; 82: 587-593.

45. Yoneyama T, Yoshida M, Ohrui T et al. Oral care reduces pneumonia in older patietns in nursing homes. J Am Geriatr Soc 2002; 50: 430-433.

46. Abe S, Ishihara K, Adachi M, Sasaki H, Tanaka K, Okuda $K$. Professional oral care reduced influenza infection in elderly. Arch Gerontol Geriatr 2006; 43: 157-164.

47. Quagliarello V, Ginter S, Han L, Van Ness P, Allore H, Tinetti M. Modifiable risk factors for nursing homeacquired pneumonia. Clin Infect Dis 2005; 40: 1-6.

48. Imsand M, Janssens J P, Auckenthaler R, Mojon P, Budtz-Jørgensen E. Bronchopneumonia and oral health in hospitalised older patients. Gerodontology 2002; 19: $66-72$.

49. Mori H, Hirasawa $H$, Oda S, Shiga H, Matsuda K, Nakamura M. Oral Care reduced incidence of ventilator associate pneumonia in ICU populations. Intensive Care Med 2006; 32: 230-236.

50. Chakraborty S. Metagenome of SARS-Cov2 patients in Shenzhen with travel to Wuhan shows a wide range of species - Lautropia, Cutibacterium, Haemophilus being most abundant - and Campylobacter explaining diarrhoea. 2020. Available online at https://osf.io/ jegwq (accessed June 2020). 